\title{
Non-Intrusive Generalized Polynomial Chaos for the Robust Stability Analysis of Uncertain Nonlinear Dynamic Friction Systems
}

Lyes Nechak $^{\mathrm{a}}$, Sébastien Berger ${ }^{\mathrm{a}}$, Evelyne Aubry ${ }^{\mathrm{a}}$

a MIPS Laboratory, University of Haute Alsace, 12 Rue des frères Lumière 68093

Mulhouse, France, lyes.nechak@uha.fr sebastien.berger@uha.fr evelyne.aubry@uha.fr, Phone : 0389336944, Fax :0389423282

Corresponding author : Sébastien Berger

\begin{abstract}
This paper is devoted to the stability analysis of uncertain nonlinear dynamic dry friction systems. The stability property of dry friction systems is known to be very sensitive to the variations of friction laws. Moreover, the friction coefficient admits dispersions due to the manufacturing processes. Therefore, it becomes necessary to take this uncertainty into account in the stability analysis of dry friction systems to ensure robust predictions of stable and instable behaviours. The generalized polynomial chaos formalism is proposed to deal with this challenging problem treated in most cases with the prohibitive Monte Carlo based techniques. Two equivalent methods presented here combine the non-intrusive generalized polynomial chaos with the indirect Lyapunov method. Both methods are shown to be efficient in the estimation of the stability and instability regions with high accuracy and high confidence levels and at lower cost compared with the classic Monte Carlo based method.
\end{abstract}

Key word: Dry friction systems, uncertain nonlinear dynamic systems, stability, generalized polynomial chaos, non-intrusive schemes, Lyapunov approach 


\section{INTRODUCTION}

Several studies have been devoted to the analysis of the dynamic behavior of dry friction systems. The stability analysis problem of this particular class of nonlinear dynamic systems occupies a major place. Indeed, a high number of studies, involving both scientists and industrialists, were carried out in order to define efficient methods for predicting instability in dry friction systems such as braking, wiping and clutch systems [1-7]. Various mechanisms have been defined to explain the self friction-induced vibration phenomenon. They are classified into two main families. The first one is related to the tribological aspects of mechanical systems and includes the stick-slip and speed dependent friction force mechanisms, while the second family is related to geometrical and structural properties and includes the so-called sprag-slip and mode coupling mechanisms [7-13]. The stick-slip and speed dependent friction force rely on changes in the friction coefficient according to the relative sliding speed between two bodies in contact. The stick-slip received greater interest. It characterizes instabilities as a consequence of a low sliding speed phenomenon caused when the static friction coefficient is higher than the dynamic coefficient. The stick slip has been shown to be relevant in modelling friction-induced instabilities such as low frequency brake vibrations for example [1], [14-19] but numerous studies have pointed out that a decrease in the friction coefficient is not sufficient to explain other types of friction-induced vibrations [10], [20-22]. In a more general setting, the tribological properties are not the unique cause of instabilities since the latter have been shown to occur even if the friction coefficient remains constant with respect to the relative sliding speed. In this context, the structural and geometrical based mechanisms have been proposed, namely the sprag-slip and mode coupling, by which friction-induced oscillations are generated with a constant friction coefficient. The Sprag slip was introduced in 1961 by Spurr who gave a purely structural 
interpretation of the self-excited friction induced vibrations [10]. A more developed theory describes instabilities as geometrically induced or kinematic constraint instability. D'Souza and Dweib show analytically that friction is responsible for the presence of a coupling term between modes which induces the destabilization of a stationary state [21]. The same conclusion appears in numerous other studies. Hoffmann proposes a two degree of freedom system in which the friction-induced vibrations are explained by a mode coupling phenomenon defined by a coalescence of the system's modes at the Hopf bifurcation point which occurs with a constant friction coefficient [23]. Numerous recent studies focus on the sprag-slip and mode coupling in frictions induced vibrations [24-27]. The friction coefficient is considered constant. In fact and in practice, the friction coefficient is a random parameter since it presents dispersions related in the general cases to the manufacturing process which yields uncertain characteristics for the contact surfaces in mechanical systems. So, for two manufactured systems of the same type, the corresponding friction coefficients are constant according to the sprag-slip and mode coupling phenomena but with different values. However, the stability property of dry friction systems is currently known to be very sensitive to even small variations of the friction coefficient. So, it is necessary to take account of the above mentioned uncertainty in the stability analysis in order to predict stability and instability behaviours with robustness. This task is an important step in the robust design framework which aims to choose suitable design parameters which ensure the best stability properties of friction systems according to known random supports of friction coefficients.

The stability analysis of nonlinear dynamic friction systems with uncertain friction coefficients can be included in the stochastic stability analysis framework. The main interest of the latter is to obtain stability assessment for dynamic systems by taking account of uncertainty (in parameters, inputs, initial conditions, etc). Different approaches have been 
proposed in this field. For uncertain linear systems, if the parameters are linear functions of the random variables modelling uncertainties, then it is only necessary to test the stability corresponding to the extreme value of the uncertain parameters [28]. For general cases, Monte Carlo methods based on sampling processes are the most useful [29-31]. These methods try to estimate probability densities using a great number of points. This consists in creating a grid of values according to the probabilistic support of uncertainties and in operating simulations and analyses for each point of the grid. In the stability analysis framework, the conditions for stability are tested for each point to determine if the system is stable for the whole distribution. This method is known to be prohibitive since it requires a high number of samples to ensure good convergence of the MC procedure and a high level of accuracy and confidence for the stability analysis. In this context, the polynomial chaos theory can be seen as an interesting alternative. The polynomial chaos concept helps to describe random functions with convergent polynomial functions series in some independent random variables with known joint density functions [32-33]. The efficiency of its intrusive and non-intrusive implementation schemes has been shown in numerous applications such as treating uncertainties in environmental and biological problems [34] and in multibody dynamic systems [35-36], flow simulation problems [37-38], sensitivity analysis [39-40], robust analysis of uncertain nonlinear dynamic systems [41] (Nechak et al, 2010), parameter estimation [42-44], controller design problems [45-46] and limit cycle analysis [47-48].

Fischer and Bhattacharya propose the generalized polynomial chaos formalism to study the stability of stochastic nonlinear dynamic systems. The main idea of their method is to transform the stochastic differential equations by means of an intrusive Galerkin projection into a deterministic set of differential equations. The Lyapunov direct method is then used to study the stability of the resulting systems [49]. Nechak et al have tested the efficiency of this 
method by using it to analyze the stability of a drum brake system with an uncertain friction coefficient governed by a uniform probabilistic law. The obtained conclusion states that the method gives an efficient tool to model and approximate the evolution of uncertainties in the state trajectories and to estimate stable intervals when the generalized polynomial chaos (GPC) approximation is suitably constructed [50]. However, the method cannot be used to predict instabilities.

The main originality in this paper lies in the two methods proposed to predict both stability and instability behaviours in uncertain nonlinear dynamic friction systems. The two methods are based on the non-intrusive generalized polynomial chaos formalism coupled with the Lyapunov indirect approach. The idea common to both methods is to express the eigenvalues of the linearized system by means of the generalized polynomial chaos expansions which are computed with a non-intrusive scheme. In the first method, a sampling based procedure is combined with the GPC model to reconstruct the probabilistic distribution of the eigenvalues. Stability is then analyzed using the indirect Lyapunov approach. In the second method, first some nonlinear optimization problems are solved to obtain the minimum and the maximum of the eigenvalues' real parts corresponding to the uncertainty support. Stability is then analyzed according to an extended Lyapunov indirect criterion. The efficiency of the proposed methods is tested in the stability analysis of a drum brake system in which the friction coefficient is a random parameter.

This paper is organized as follows. The Lyapunov indirect approach for stability analysis of nonlinear dynamic systems is recalled in Section 2. The generalized polynomial chaos theory is presented in Section 3, then used to formulate the stability analysis of uncertain nonlinear systems in Section 4. The application of the proposed methods and the corresponding results are shown in Section 5, followed by conclusions at the end of the paper. 


\section{STABILITY ANALYSIS OF NONLINEAR DYNAMIC SYSTEMS WITH THE INDIRECT LYAPUNOV APPROACH}

Consider a nonlinear dynamic system with a real uncertain parameter $\mu$ with a known density function.

$$
\dot{\mathbf{x}}=\mathbf{f}(\mathbf{x}, \mu)
$$

with $\mathbf{f}$ is a smooth nonlinear vector field, $\mathbf{x} \in \mathbf{R}^{n}$ is the state vector.

Suppose, without lose of generality, that the origin is an equilibrium point for the system (1). Moreover, it is not affected by the uncertainty of the $\mu$ parameter. So the origin is assumed to be the equilibrium in the whole distribution of $\mu$. The uncertain Jacobian matrix corresponding to the system (1) is given by

$$
\mathbf{A}(\mu)=\left(\frac{\mathrm{d} \mathbf{f}(\mathbf{x}, \mu)}{\mathrm{d} \mathbf{x}}\right)_{\mathbf{x}=0}
$$

The stability of the origin in the system (1) is analyzed following the Lyapunov's indirect method using the Jabobian matrix [51] combined with a Monte Carlo type procedure. It can be summarized in three main steps:

1. Generate samples following the probabilistic support of parameter $\mu$.

2. Compute, for each sample, the corresponding eigenvalue of matrix $\mathbf{A}$.

3. Analyze the stability, for each sample, using the indirect Lyapunov method. 
In the stability analysis, one crucial problem is to find the Hopf bifurcation point. The point $\left(\mathbf{0}, \mu_{0}\right)$ is said to be the Hopf bifurcation point of the system (1) if the following conditions are fulfilled.

- $\left.\operatorname{Re}\left(\boldsymbol{\lambda}_{\text {center }}(\mu)\right)\right|_{(\mathbf{x}, \mu)=\left(0, \mu_{0}\right)}=\mathbf{0}$

- $\left.\operatorname{Re}\left(\boldsymbol{\lambda}_{\text {non-center }}(\mu)\right)\right|_{(\mathbf{x}, \mu)=\left(\mathbf{0}, \mu_{0}\right)} \neq \mathbf{0}$

- $\left.\frac{\mathrm{d}}{\mathrm{d} \mu}(\lambda(\mu))\right|_{\mu=\mu_{0}} \neq \mathbf{0}$

At the bifurcation point $\left(\mathbf{0}, \mu_{0}\right)$, the Jacobian matrix (2) has at least a pair of purely imaginary eigenvalues $\lambda_{\text {center }}(\mu)$ while the other eigenvalues $\lambda_{\text {non-center }}(\mu)$ remain non-zero real parts. The condition (5) called transversal condition, implies a transversal or a non-zero speed crossing of the imaginary axis [51].

As mentioned in the introduction, the sampling based method is known to be prohibitive since it requires a high number of samples to ensure reasonable accuracy with high confidence. The resulting computing cost is exorbitant since the system's eigenvalues must be calculated for each sample, an operation which is difficult, especially for systems with numerous degrees of freedom. Therefore, the generalized polynomial chaos formalism can be used instead of the classic Monte Carlo procedure. This theory is described in the following section. 


\section{GENERALIZED POLYNOMIAL CHAOS THEORY}

The generalized polynomial chaos establishes a separation between the stochastic components of a random function and its deterministic components. Here is the mathematical framework of this approach. From the Wiener theory and the generalizes Cameron-Martin theorem, any second order random process $x$ can be expanded in a convergent (in the mean square sense) polynomial function series as:

$$
x=\sum_{j=0}^{\infty} \bar{x}_{j} \phi_{j}(\xi)
$$

$\xi$ is a vector of $\mathrm{d}$ independent random variables with known joint density function $W(\xi)$, $\bar{x}_{j}$ are the stochastic modes of the random process $x$ and $\phi_{j}$ are orthogonal polynomial functions $\phi_{j}$ satisfying the orthogonality relation (7).

$$
\left\langle\phi_{i}, \phi_{j}\right\rangle=\int \phi_{i}(\xi) \phi_{j}(\xi) W(\xi) d \xi= \begin{cases}0 & i \neq j \\ \left\langle\phi_{i}, \phi_{i}\right\rangle & i=j\end{cases}
$$

$\langle$.$\rangle being the internal product operator.$

In practice, generalized polynomial chaos expansion (6) is truncated to a finite number of terms.

$$
x \approx \sum_{j=0}^{P} \bar{x}_{j} \phi_{j}(\xi)
$$

The truncation order $P$ is shown to be dependent on the generalized polynomial chaos $r$ of the polynomial functions $\phi_{j}$ and the stochastic dimension $d$ denoting the number of uncertain parameters. 


$$
P=\frac{(d+r) !}{d ! r !}-1
$$

Then, computing $x$ is transformed into the problem of finding the coefficients $\bar{x}_{j}$ of its truncated expansion. The intrusive and non intrusive approaches are defined to calculate these coefficients called stochastic modes. The non-intrusive approach is shown to be more efficient since it only needs simulations corresponding to particular samples of the random variables and it needs no modifications of the stochastic model, contrary to the intrusive approach. The latter consists in operating a Galerkin projection to generate, from the stochastic model, a set of deterministic coupled equations which are unfortunately difficult to implement, especially for complex nonlinear models. That is why only the non intrusive approach is considered in this paper.

\section{STABILITY ANALYSIS USING THE GENERALIZED POLYNOMIAL CHAOS APPROACH AND THE LYAPUNOV INDIRECT METHOD}

Two methods based on the generalized polynomial chaos theory and the Lyapunov indirect approach are proposed to deal with the stability analysis of the dynamic system (1) in which $\mu$ is the only uncertain parameter. Thus, $d=1$ and $P=r$. The $\mu$ parameter is supposed to be governed by a uniform distribution function within a given interval $[a, b]$.

As parameter $\mu$ is uncertain, all the eigenvalues, $\lambda_{i}(i=1, \ldots, n)$ of the Jacobian matrix (2) are also random functions. According to the Askey scheme [37-38], the Legendre polynomials are the best suited to deal with uniform uncertainties, so the random eigenvalues are given by:

$$
\lambda_{i}(\xi) \approx \sum_{j=0}^{P} \bar{\lambda}_{i, j} L_{j}(\xi)
$$


Here $\xi$ is distributed uniformly within the orthogonality interval $[-1,1]$ of the Legendre polynomials. It models the uncertainty of parameter $\mu$. Therefore, $\mu=\bar{\mu}_{0}+\bar{\mu}_{1} \xi$ with $\bar{\mu}_{0}$ is the mean value and $\bar{\mu}_{1}$ is a convenient constant.

The step common to the proposed methods is to compute the stochastic modes $\bar{\lambda}_{i, j}$. The nonintrusive spectral projection (NISP) or the regression technique can be used to compute the stochastic modes $\bar{\lambda}_{i, j}$.

The NISP technique exploits the orthogonality property of the Legendre polynomials $L_{j}(\xi)$ to express $\bar{\lambda}_{i, j}$ from (10) as follows.

$$
\bar{\lambda}_{i, j}=\left(1 /\left\langle L_{j}, L_{j}\right\rangle\right) \int_{-1}^{1} \lambda_{i}(\xi) L_{j}(\xi) W(\xi) d \xi
$$

Numerical techniques are used for integral computing, such as the Simpson or the Gauss collocation methods. With the latter, expression (11) can be approximated with formula (12).

$$
\bar{\lambda}_{i, j} \approx\left(1 /\left\langle L_{j}, L_{j}\right\rangle\right) \sum_{k=1}^{Q} \lambda_{i}\left(\xi^{(k)}\right) L_{j}\left(\xi^{(k)}\right) W^{(k)}
$$

where $\xi^{(k)}$ and $W^{(k)}, k=1, \ldots, Q$ are given by the well known Gauss collocation points and their corresponding weights.

Once the stochastic modes have been obtained, the stability analysis of the system (1) can be performed with one of the following proposed methods. 


\section{1. $\quad$ First method}

The first method considers a sampling process on the probabilistic distribution of the random variable $\xi$ and reconstructs the eigenvalues using the Legendre polynomial chaos expansion (10). The stability is then analyzed with the classic Lyapunov indirect criteria [51]. So, for a given sample $\xi^{(k)}$ the origin is:

- Asymptotically stable if :

$$
\forall i \in\{1, \ldots, n\} / \operatorname{Re}\left(\lambda_{i}\left(\xi^{(k)}\right)\right)<0
$$

- Unstable if :

$$
\exists i \in\{1, \ldots, n\} / \operatorname{Re}\left(\lambda_{i}\left(\xi^{(k)}\right)\right)>0
$$

- With no conclusion on the stability if :

$$
\exists j \in\{1, \ldots, n\}, \forall i \neq j / \operatorname{Re}\left(\lambda_{j}\left(\xi^{(k)}\right)\right)=0 \wedge \operatorname{Re}\left(\lambda_{i}\left(\xi^{(k)}\right)\right)<0
$$

The origin is asymptotically stable (or unstable) on the whole distribution with some confidence level depending on the number of samples $N$, if condition (13) (or condition (14)) is verified for all the generated samples $\xi^{(k)}$. The main advantage of this method is that it helps to overcome the computational difficulties of the classic Monte Carlo method. With the latter, the stability analysis passes through solving the characteristic equation of the system for each sample to obtain the eigenvalues. This operation is too costly and difficult especially for systems with numerous degrees of freedom. With the Legendre polynomial chaos, no characteristic equation needs to be solved. To obtain the eigenvalues, only the Legendre polynomials need to be evaluated at the corresponding samples. 


\subsection{Second method}

The first method uses a sampling based principle. To avoid this operation, the stability analysis can be performed by solving a certain number of optimization problems (16) to obtain the maxima and minima of the real part of the system's eigenvalues then to use an extended version of the Lyapunov indirect criteria.

$$
\left\{\begin{array}{c}
\max / \min \left(\operatorname{Re}\left(\lambda_{i=1, \ldots, n}(\xi)=\sum_{j=0}^{P} \bar{\lambda}_{i, j} L_{j}(\xi)\right)\right) \\
\xi \in[-11]
\end{array}\right.
$$

The stability on the whole distribution of the parameter $\mu$ can be analyzed by extending the Lyapunov indirect criteria (13), (14) and (15). So for $\xi \in[-1,1]$ the origin is:

- Asymptotically stable if:

$$
\forall i \in\{1, \ldots, n\}, \max _{\xi \in[-1,1]}\left(\operatorname{Re}\left(\lambda_{i}(\xi)\right)\right)<0
$$

- Unstable if:

$$
\exists i \in\{1, \ldots, n\}, \min _{\xi \in[-1,1]}\left(\operatorname{Re}\left(\lambda_{i}(\xi)\right)\right)>0
$$

- With no conclusion on the stability if:

$$
\begin{gathered}
\exists i \in\{1, \ldots, n\}, \forall j\{1, \ldots, n\} / \max _{\xi \in[-11]}\left(\operatorname{Re}\left(\lambda_{i}(\xi)\right)\right) \geq 0 \\
\wedge \min _{\xi \in[-11]}\left(\operatorname{Re}\left(\lambda_{j}(\xi)\right)\right) \leq 0
\end{gathered}
$$


The dichotomy principle can be used to suppress uncertainty in the conclusion corresponding to (19). The main idea is to divide the Legendre Support into two equal intervals at each time; (19) is verified until the separation of the stable regions from the unstable ones.

The accuracy of both proposed methods depends on the accuracy of the Legendre polynomial chaos based model (10). So, it is important to set an appropriate order $P$ which ensures an acceptable level of accuracy.

\section{APPLICATION AND RESULTS}

In order to test the efficiency of the proposed methods, they are used to analyze the stability of a two degree of freedom model representing a drum brake system subject to an uncertain friction coefficient. The main aim is to separate the stable and unstable regions corresponding to a known probabilistic dispersion of the friction coefficient. So, the system considered is described. The non-intrusive generalized polynomial chaos based methods coupled with the Lyapunov indirect method are then applied to the stability analysis problem.

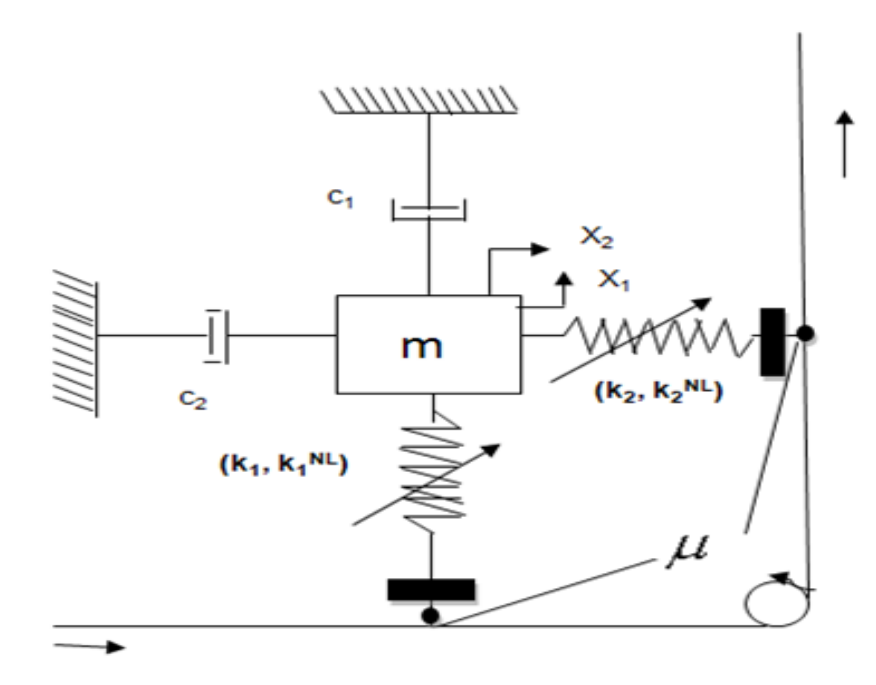

Figure 1. Mechanical model 
The system shown in the previous figure was defined by Hulten in his study of squeal vibration of brake systems [52] then used in many other studies as the definition of a robust damping factor in self exciting mechanisms [53] and the robust analysis of the dynamic behaviour of friction systems [50]. This system is a two degree of freedom model which has been shown to be sufficient to study mode coupling instability [26].

As shown in Figure 1, the system considered is composed of a mass $m$ held against a moving band; the contact between the mass and the band is modelled by two plates supported by two different springs. For the sake of simplicity, it is assumed that the mass and band surfaces are always in contact. This assumption may be due to a preload applied to the system. The contact can be expressed by two cubic stifnesses. Damping is integrated as shown in Figure 1. The friction coefficient at contact is assumed to be constant and the band moves at a constant velocity. Then it is assumed that the direction of the friction force does not change because the relative velocity between the band speed $\dot{X}_{1}$ or $\dot{X}_{2}$ is assumed to be positive. The tangential force $F_{T}$ due to the friction contact is assumed to be proportional to the normal force $F_{N}$ as given by Coulomb's law: $F_{T}=\mu F_{N}$. The resulting motion equation can be expressed in the state space, as shown in Hultén and Sinou studies [50], [53], [26].

$$
\begin{aligned}
\dot{\mathbf{x}}(t) & =\mathbf{f}(\mathbf{x}(t), \mu) \\
& =\mathbf{A}(\mu) \mathbf{x}(t)+\mathbf{f}_{\mathbf{N L}}(\mathbf{x}(t), \mu)
\end{aligned}
$$


where $\quad \mathbf{x}(t)=\left[\begin{array}{llll}x_{1}(t) & x_{2}(t) & x_{3}(t) & x_{4}(t)\end{array}\right]^{T}$ with $\quad x_{1}(t)=X_{1}(t), \quad x_{2}(t)=\dot{X}_{1}(t)$, $x_{3}(t)=X_{2}(t), \quad x_{4}(t)=\dot{X}_{2}(t), \quad \mathbf{A}(\mu)=\left[\begin{array}{cccc}0 & 1 & 0 & 0 \\ -\omega_{1}^{2} & -\eta_{1} \omega_{1} & \mu \omega_{2}^{2} & 0 \\ 0 & 0 & 0 & 1 \\ -\mu \omega_{1}^{2} & 0 & -\omega_{2}^{2} & -\eta_{2} \omega_{2}\end{array}\right], \quad$ and $\mathbf{f}_{\mathbf{N L}}(\mathbf{x}(t), \mu)=\left[\begin{array}{c}0 \\ -\varphi_{1}^{N L} x_{1}^{3}(t)+\mu \varphi_{2}^{N L} x_{3}^{3} \\ 0 \\ -\mu \varphi_{1}^{N L} x_{1}^{3}(t)-\varphi_{2}^{N L} x_{3}^{3}(t)\end{array}\right]$

with $\eta_{i}=c_{i} / \sqrt{m k_{i}}$ the relative damping coefficients, $\omega_{i}=\sqrt{k_{i} / m}$ the natural pulsations and $\varphi_{i}^{N L}=k_{i}^{N L} / m$ for $i=1,2$. For numerical application: $m=1 \mathrm{~kg}, \quad \omega_{1}=2 \pi \times 100 \mathrm{rad} . \mathrm{s}^{-1}$, $\omega_{2}=2 \pi \times 75 \mathrm{rad} . \mathrm{s}^{-1}, \eta_{1}=\eta_{2}=0.02, \varphi_{1}^{N L}=\omega_{1}^{2}, \varphi_{2}^{N L}=0$.

The friction coefficient $\mu$ is an uncertain constant parameter supposed to be driven by a uniform distribution law within the interval $[0,0.5]$.

It can be noted that the origin is an equilibrium point of the state equation (20). Moreover, the uncertainty of the friction coefficient does not affect the equilibrium position. Now, it will be interesting to analyze the origin stability by taking the dispersion of the friction coefficient into account.

\subsection{Stability analysis with a Monte Carlo (MC) type procedure}

The stability of the friction system equilibrium is investigated using a classic MC type method. The friction coefficient is assumed to have dispersions within the interval $\left[\begin{array}{ll}0 & 0.5\end{array}\right]$. 
The eigenvalues $\lambda_{i}(i=1, \ldots, n)$ of the linearized system can be found by solving the characteristic equation (21) for a set of samples generated from the probabilistic support of the friction coefficient. 10,000 samples are generated in a manner to ensure a high confidence level (99\%) with high accuracy (1\%).

$$
\operatorname{det}(\mathbf{A}(\mu)-\lambda \mathbf{I})=0
$$

Two couples of conjugate eigenvalues are obtained for the fixed interval of dispersion. The evolutions of their real and imaginary parts are plotted against the values of the friction coefficient in the figure 2 .
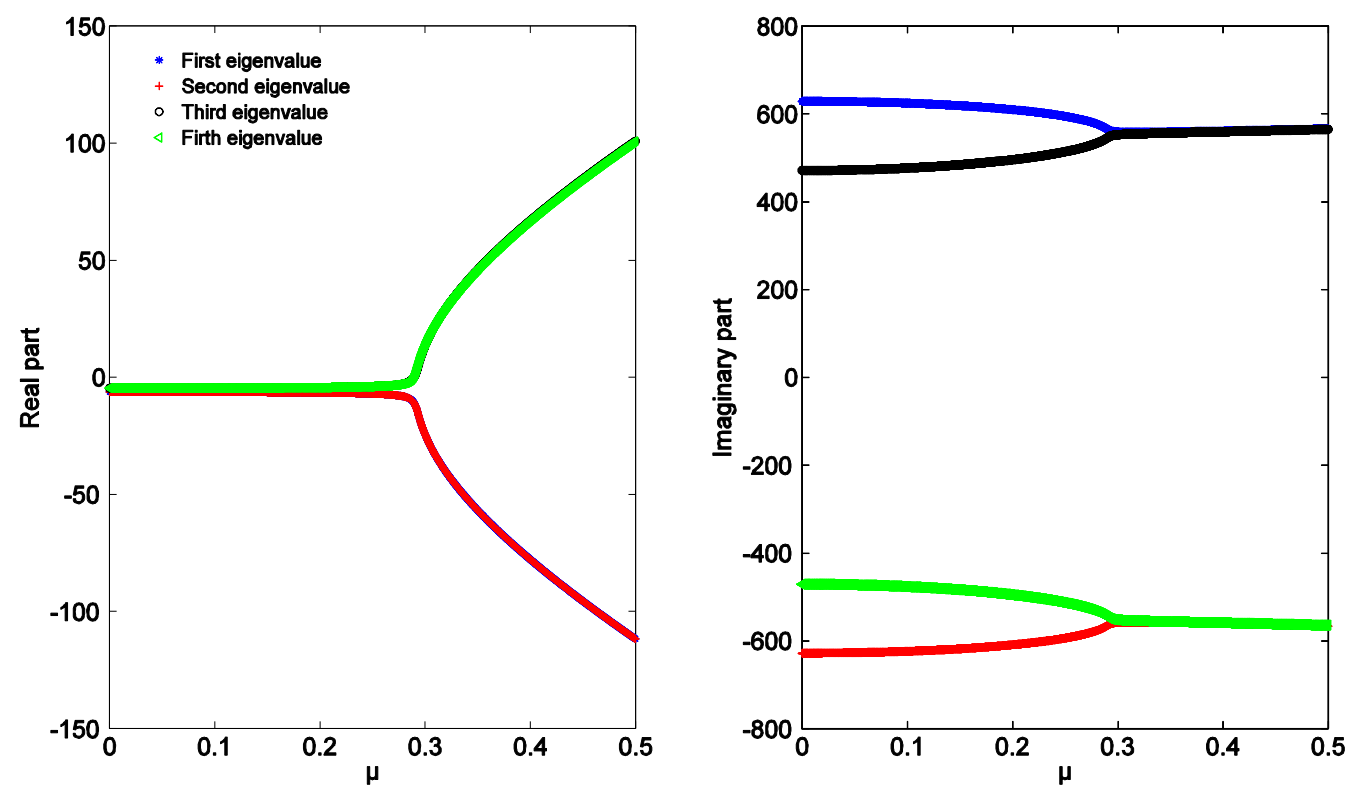

Figure 2. Eigenvalues of the system (20) according to the friction coefficient

As long as the real parts of all the eigenvalues remain negative, the origin system (20) is asymptotically stable. When at least one of the eigenvalues has a positive real part, the origin is unstable. The imaginary parts of these eigenvalues represent instability frequencies. The 
Hopf bifurcation point corresponding to a couple of eigenvalues with purely imaginary parts is obtained at $\mu=0.2894$.

As mentionned previously, computational difficulties are caused by the MC method which, consequently, becomes unsuitable for complex nonlinear systems with numerous degrees of freedom. Two methods are proposed to circumvent the computational difficulties of the classic MC type method.

\subsection{Application of the generalized polynomial chaos coupled with the Lyapunov indirect method}

Contrary to the classic MC method used previously, the distribution of the eigenvalues of the linearized approximation of system (20) is reconstructed by coupling an MC type method with model (10). The latter must be determined in a manner to ensure an accurate approximation of the evolution of the eigenvalues which defines the stability. So, different truncation orders $P$ are considered for model (10). The evolutions of the real and imaginary parts of the system's eigenvalues with respect to the uncertain friction coefficient are plotted respectively in Figures 3 and 4. They are obtained with 10,000 samples and compared to the classic MC method using the same number of samples in order to have the same confidence and accuracy parameters. From Figures 3 and 4 , model (10) with $P=30$ gives the best approximation comparing with those obtained with $P=25$ and $P=20$. From a statistical point of view, the distribution laws constructed with model (10) and shown in Figure 5, are correct, compared to the reference laws given by the MC method. However, the crucial point is the estimation of the Hopf bifurcation zone. At the Hopf bifurcation point, the system has a pair of purely imaginary eigenvalues while the other eigenvalues remain stable. So, near the 
Hopf bifurcation point, the system loses its asymptotic stability and becomes unstable for an infinitesimal perturbation. The Hopf bifurcation point is estimated with model (10) using the different orders. The results are illustrated in table 1.
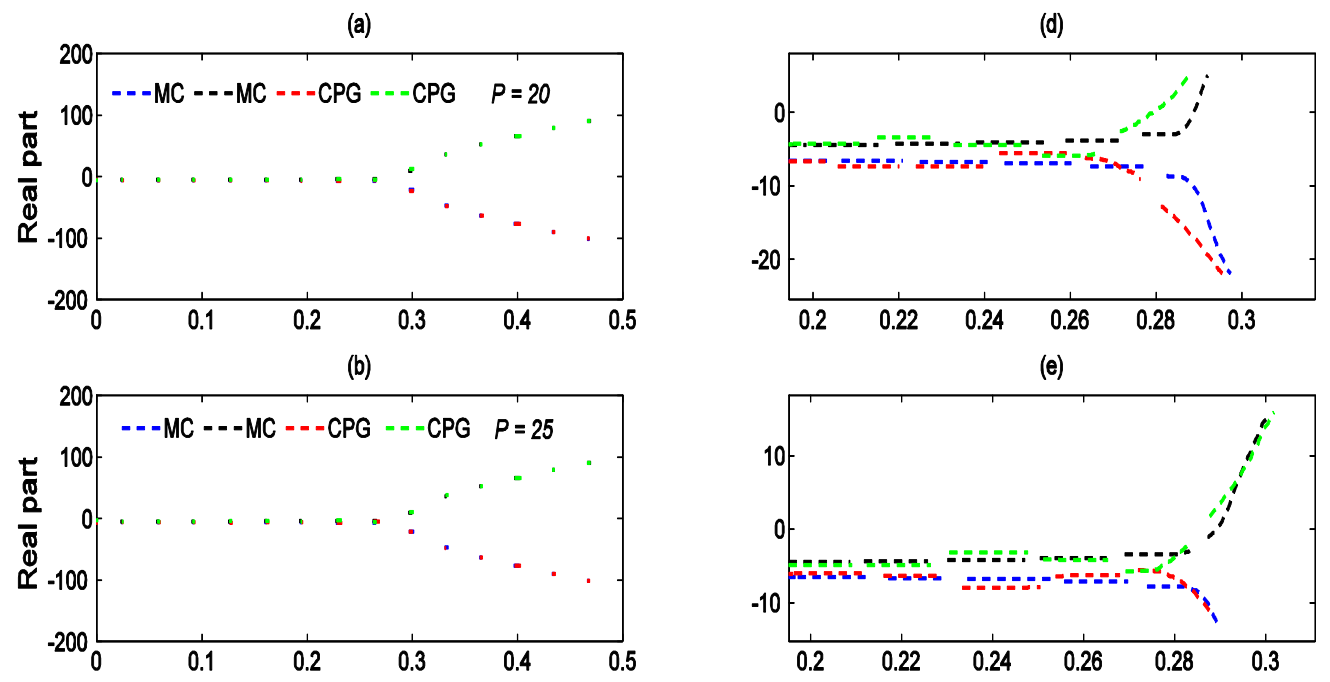

(e)
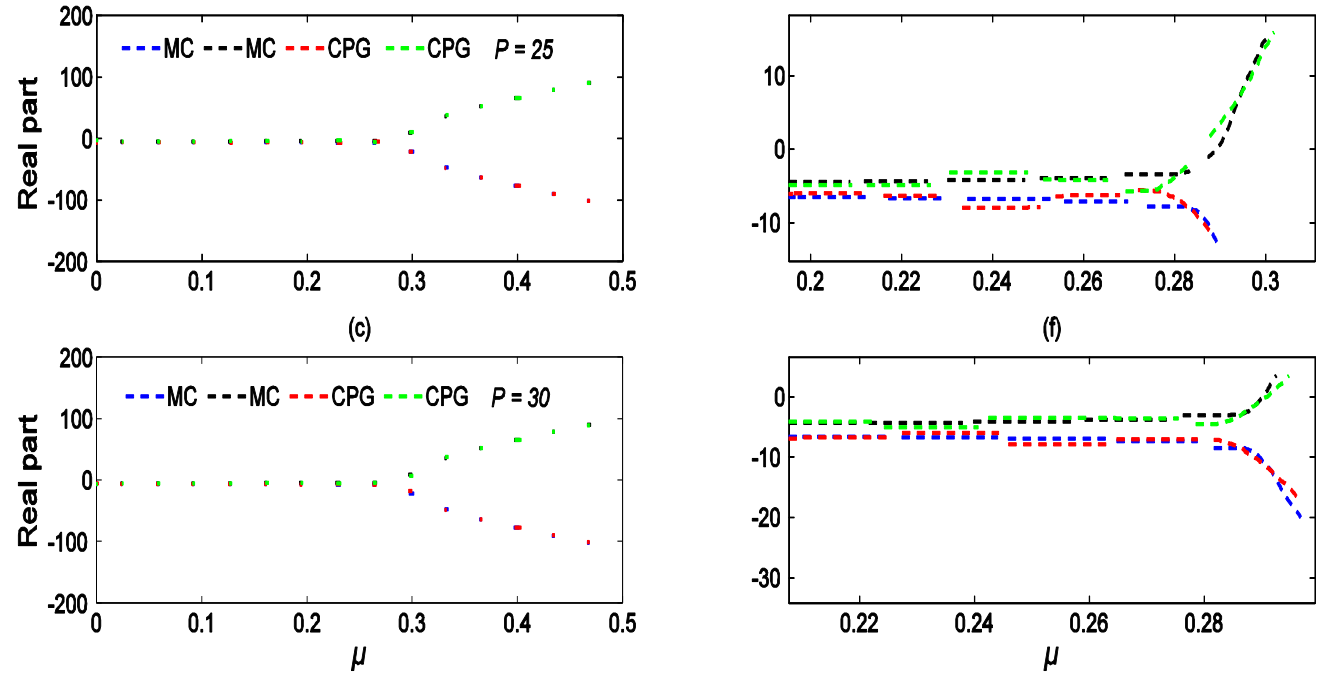

Figure 3. $\{(a),(b),(c)\}$ : Evolution of the real parts of the system's eigenvalues with respect to the friction coefficient- $\{(\mathrm{d}),(\mathrm{e}),(\mathrm{f})\}$ : zoom on the Hopf bifurcation zones in $\{(\mathrm{a}),(\mathrm{b}),(\mathrm{c})\}$ respectively 

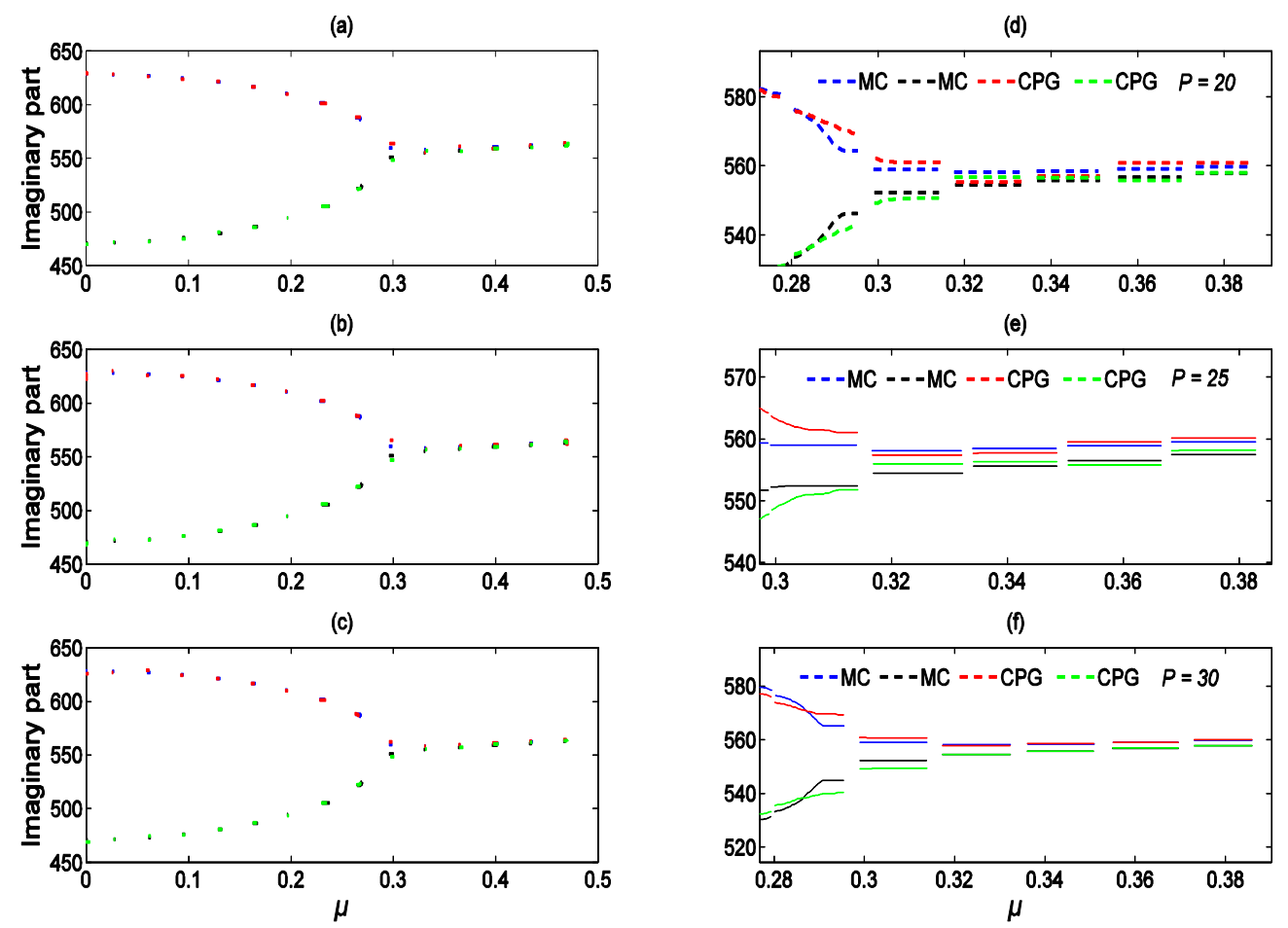

Figure 4. $\{(\mathrm{a}),(\mathrm{b}),(\mathrm{c})\}$ : Evolution of the imaginary parts of the system's eigenvalues with respect to the friction coefficient- $\{(\mathrm{d}),(\mathrm{e}),(\mathrm{f})\}$ : zoom on the Hopf bifurcation zones in $\{(a),(b),(c)\}$ respectively 

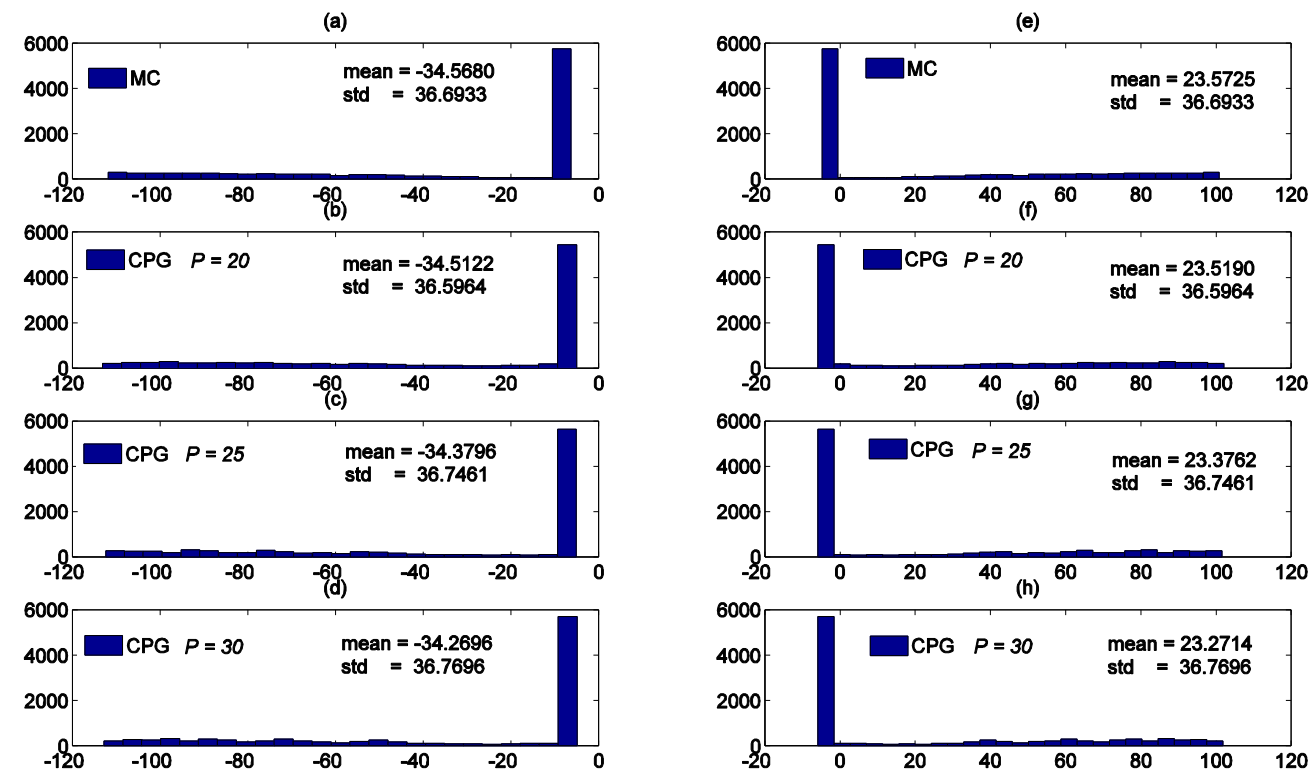

Figure 5. Probabilistic distribution of the real parts of the system's eigenvalues- $\{(\mathrm{a}),(\mathrm{b}),(\mathrm{c})$, (d) $\}$ :Histograms of the real parts of the first pair of eigenvalues- $\{(\mathrm{e}),(\mathrm{f}),(\mathrm{g}),(\mathrm{h})\}$ : Histograms of the real parts of the second pair of eigenvalues

\begin{tabular}{|l|l|l|l|}
\hline $\begin{array}{l}\text { Reference } \\
\text { value with } \\
\text { MC }\end{array}$ & LePC & LePC & LePC \\
\hline$\mu=0.2894$ & 0.2778 & 0.2853 & 0.2893 \\
\hline
\end{tabular}

Table 1. Estimation of the Hopf bifurcation point with model (10) coupled with an MC method

Model (10) with $P=30$ gives the best estimation of the Hopf bifurcation point with the smallest relative error $(0.03 \%)$. The levels of errors with $P=25$ and $P=20$ are higher. In table 2, the stability proportion is estimated using model (10) with a confidence equal to $99 \%$ and an accuracy near $1 \%$. The results obtained show the high performance of the model used. 
Indeed, it is shown that the Legendre polynomial expansion used estimates the proportion of stability with the same confidence interval as the MC method.

\begin{tabular}{|l|l|l|}
\hline Methods & MC & LePC $(P=30)$ \\
\hline Confidence intervals $(\%)$ & $57.46 \pm 1.28$ & $57.56 \pm 1.28$ \\
\hline
\end{tabular}

Table 2. Estimation of the stability proportion with confidence intervals

\subsection{Stability analysis by the optimization of the generalized polynomial chaos models}

The first method, presented in sub-Section 5.2, has shown great efficiency compared to the classic MC technique. The same level of confidence and accuracy is obtained at a lower cost. The second method proposed here does not consider the sampling based principle. The stability analysis is reduced to solving some optimization problems defined by (16). The conclusion on stability is given according to criteria (17), (18) and (19).

Consider the optimization problem (16) with $P=30$. The functions to be optimized are the real parts of the system's eigenvalues. The random variable describing the uncertainty $\xi$ is the decision variable of the optimization problem. The descent gradient based algorithms implemented in Optimtool Matlab Toolbox are used to search for the minima and maxima. The results are given in table.3. 


\begin{tabular}{|l|l|l|}
\hline Reference value & Max & Min \\
\hline $\operatorname{Re}\left(\lambda_{1,2}\right)$ & -4.892 & -113.089 \\
\hline $\operatorname{Re}\left(\lambda_{3,4}\right)$ & 102.160 & -6.040 \\
\hline
\end{tabular}

Table 3. Estimation of the minima and maxima of the real parts of the system's eigenvalues for $\mu$ within $[0,0.5]$

There are two pairs of complex conjugate eigenvalues $\lambda_{1,2}$ and $\lambda_{3,4}$. The first pair is stable since the maximum of the corresponding real parts is negative. The maximum of the real parts corresponding to the second pair is positive while its minimum is negative. So, according to criterion (19), no conclusion can be given on the stability of the origin.

The dichotomy principle is used to separate stable regions and unstable regions. It consists in the division of the uncertainty interval into two smaller intervals each time the situation defined by (19) arises. The same analysis procedure is then followed. The results of the first iteration are shown in tables 4 and 5.

\begin{tabular}{|l|l|l|}
\hline Reference value & Max & Min \\
\hline $\operatorname{Re}\left(\lambda_{1,2}\right)$ & -4.892 & -8.114 \\
\hline $\operatorname{Re}\left(\lambda_{3,4}\right)$ & -2.884 & -6.040 \\
\hline
\end{tabular}

Table 4. Estimation of the minima and maxima of the real parts of the system's eigenvalues for $\mu$ within [0 0.25$]$ 


\begin{tabular}{|l|l|l|}
\hline Reference value & Max & Min \\
\hline $\operatorname{Re}\left(\lambda_{1,2}\right)$ & -8.114 & -113.089 \\
\hline $\operatorname{Re}\left(\lambda_{3,4}\right)$ & 102.160 & -2.884 \\
\hline
\end{tabular}

Table 5. Estimation of the minima and maxima of the real parts of the system's eigenvalues for $\mu$ within [0.25 0.5$]$

In the interval $[0,0.25]$, all the maxima of the real parts of the eigenvalues are negative. This means that the origin is asymptotically stable, while in the interval $[0.25,0.5]$, no conclusion can be made. The dichotomy principle must be iterated. Performing this operation 15 times helps to systematically obtain the total zone of stability and instability separated by the Hopf bifurcation point which is obtained equal to 0.2893 . The total number of calculations is given by $6,000(=15 \times 400), 400$ being the number of iterations used to search for the minima and maxima. The number is lower than the one $(10,000)$ used with the first method (the GPC coupled with the MC technique and the indirect Lyapunov criteria). Note that it not possible to carry out a comparison between the first and second methods in terms of computing costs. This is due to the system which is simple, and so the resulting computing costs are not so significant.

In practice, the designers are confronted with the problem of determining a set of parameters for which the designed system presents good properties of stability. There are two families of parameters: a family of controllable parameters thanks to the increasingly precise manufacturing equipment and another family of non-controllable parameters due to their randomness. This is the case for the friction coefficient. The main idea is to use the 
generalized polynomial chaos formalism coupled with the Lyapunov indirect method to determine the set of controllable parameters which ensure optimal proportions of stability with high confidence and accuracy levels despite of the uncertainty of the uncontrollable parameters. For the system used, the friction coefficient is the only random parameter distributed uniformly within $[0,0.5]$. The damping coefficients are considered as the controllable parameters. So, the stability proportions are estimated using the first method (i.e. the generalized polynomial chaos model and the classic MC based method with the Lyapunov indirect approach). The results are compared to the referential results obtained with the classic MC method. The results of both methods agree, as illustrated in figures 6 and 7 .

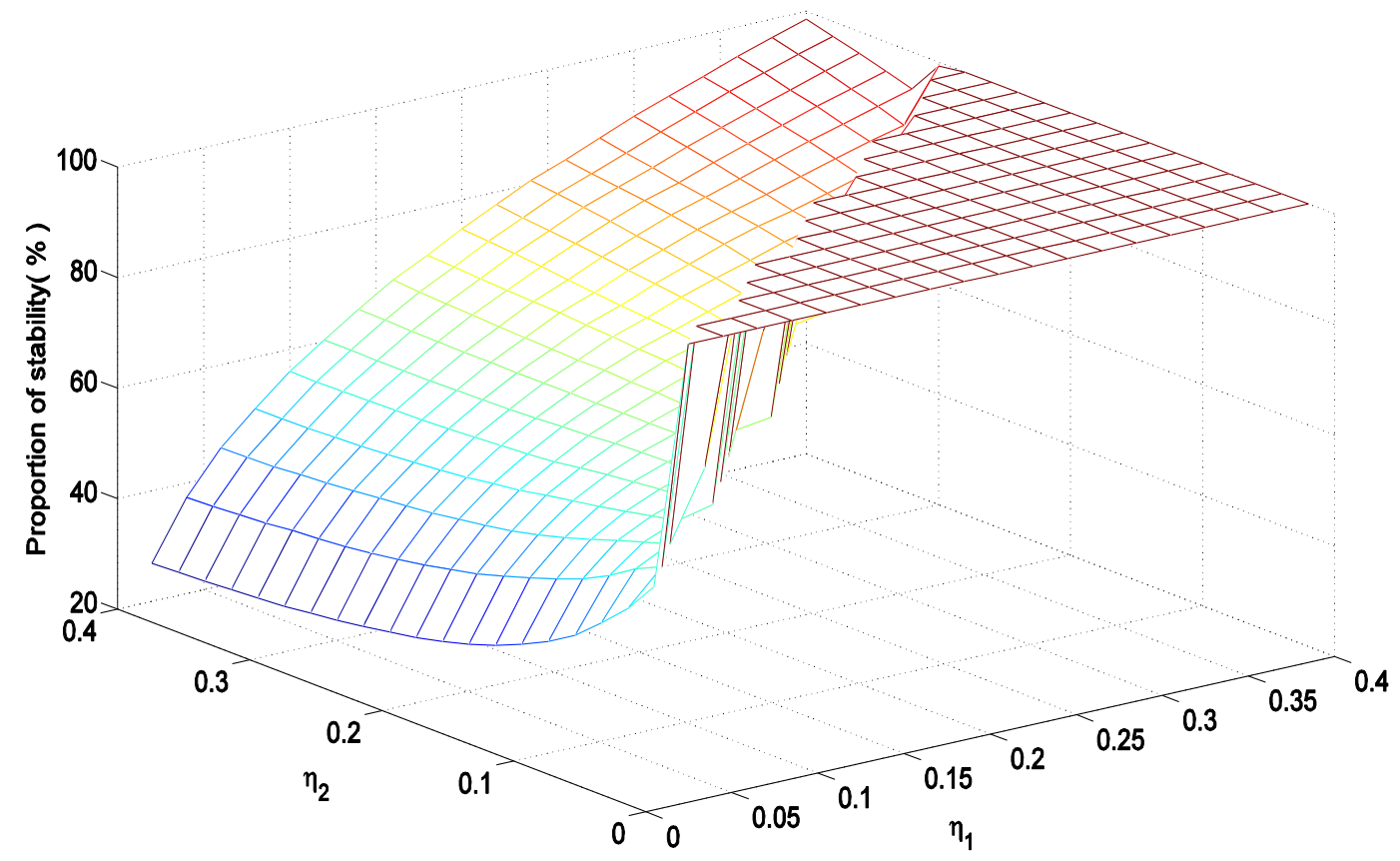

Figure 6. The stability proportions corresponding to a friction coefficient distributed uniformly within $[0,0.5]$ estimated with the first method (generalized polynomial chaos based model coupled with the MC method). 


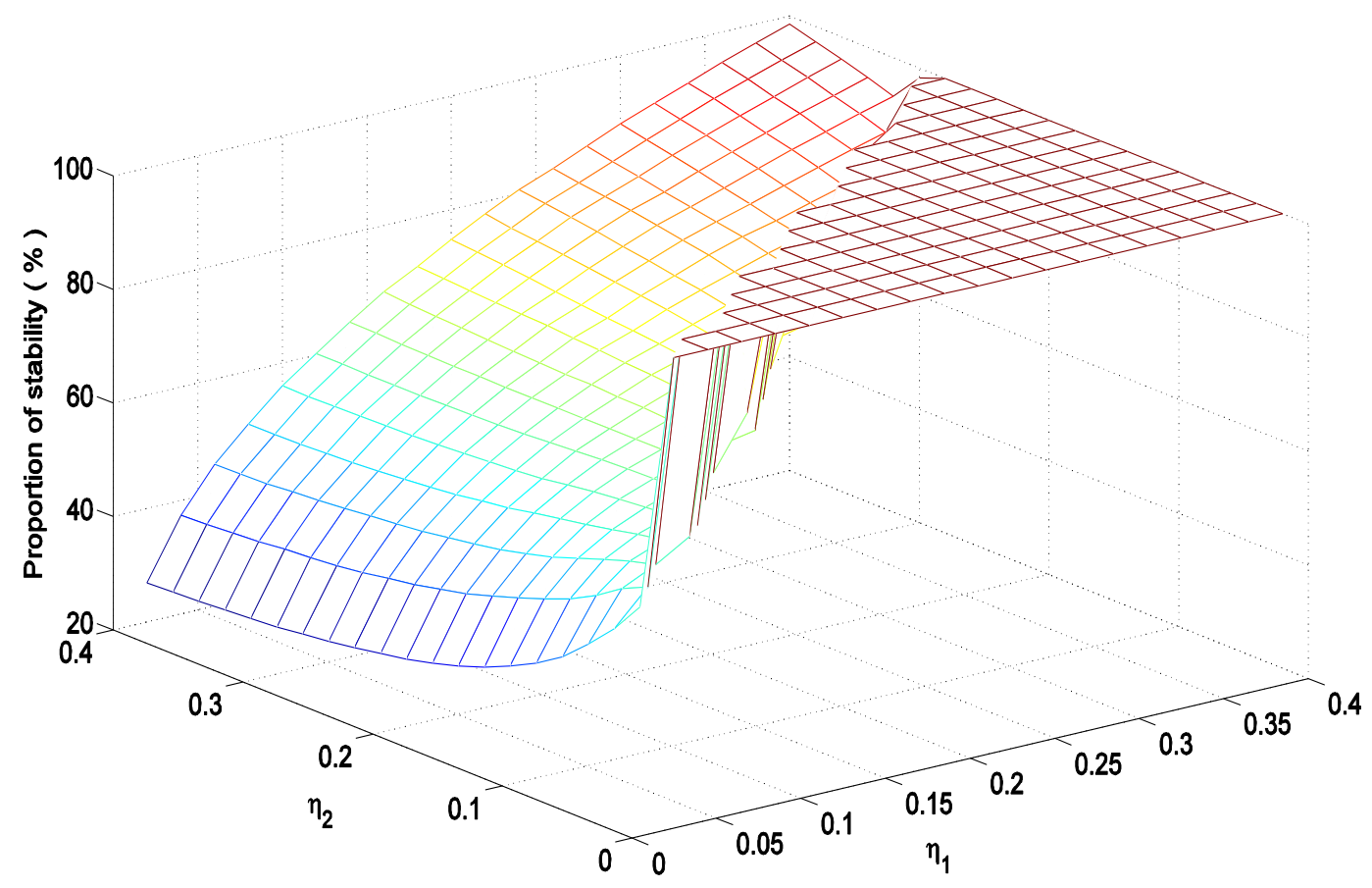

Figure 7. The stability proportions corresponding to a friction coefficient distributed uniformly within $[0,0.5]$ estimated with the classic Monte Carlo method.

\section{CONCLUSION}

Two methods have been proposed to deal with the stability of nonlinear dynamic friction systems with uncertain friction coefficients. Both methods are based on the generalized polynomial chaos formalism coupled with the Lyapunov indirect method. The main idea is to build a generalized polynomial chaos representation to model the evolution of the eigenvalues. This representation is computed using a non-intrusive approach. The first method uses a sampling based principle to reconstruct the distributions of eigenvalues. This method is shown to be of a lower cost than the classic MC method. The second method helps to avoid the use of the sampling procedure by resolving some nonlinear optimization problems. The generalized polynomial chaos representations are the objective functions which must be 
optimized in order to find the minima and maxima of the eigenvalues real parts. Stability is then analyzed according to the generalized Lyapunov indirect criteria. The efficiency of the proposed methods has been shown in the stability analysis of a brake system subject to an uncertain friction coefficient. The main aim was to show the feasibility of the proposed methods. No exhaustive comparison between the proposed methods has been performed, as the system used is too simple for a significant comparison. This point is the aim of the research work in progress in which a complex industrial friction system is considered for the evaluation of both methods.

\section{ACKNOWLEDGMENTS}

The authors are grateful for the financial support of the French National Research Agency through the Young Researcher Program ANR-07-JCJC-0059-01-CSD2

\section{REFERENCES}

[1] H. Ouyang, J.E. Mottershead, Unstable travelling waves in the friction-induced vibration of discs. Journal of Sound and Vibration 248 (2001) 768-779.

[2] J.J. Sinou, O. Dereure, F. Mazet, F. Thouverez, L. Jezequel, Friction-induced vibration for an aircraft brake system-Part 1: Experimental approach and stability analysis. International Journal of Mechanical Sciences 48 (2006) 536-554.

[3] J.J. Sinou, F. Thouverez, L. Jezequel, O. Dereure, F. Mazet, Friction-induced vibration for an aircraft brake system-Part 2: Non-linear dynamics. International Journal of Mechanical Sciences 48 (2006) 555-567. 
[4] C. Chevennement-Roux, T. Dreher, P. Alliot, E. Aubry, J. P. Lainé, L. Jézéquel, Flexible wiper system dynamic instabilities: modeling and experimental validation. Experimental Mechanics 47 (2007) 201-210.

[5] B. Hervé, J.-J. Sinou, H. Mahé, L. Jézéquel, Extension of the destabilization paradox to limit cycle amplitudes for a nonlinear self-excited system subject to gyroscopic and circulatory actions. Journal of Sound and Vibration 323 (2009) 944-973.

[6] G. Fritz, J.-J. Sinou, J.-M. Duffal, L. Jézéquel, Investigation of the relationship between damping and mode coupling patterns in case of brake squeal. Journal of Sound and Vibration 307 (2007) 591-609.

[7] N. Coudeyras, S. Nacivet, J.-J. Sinou, Periodic and quasi periodic solutions for multiinstabilities involved in brake squeal. Journal of Sound and vibration 328 (2009) 520-540.

[8] R.A. Ibrahim, Friction-induced vibration, chatter, squeal and chaos: Part I- Mechanics of contact and friction. ASME Applied Mechanics Reviews 47 (1994) 209-226.

[9] R.A. Ibrahim, Friction-induced vibration, chatter, squeal and chaos: Part II- Dynamics and modeling. ASME Applied Mechanics Reviews 47 (1994) 227-253.

[10] R.T. Spurr, A Theory of Brake Squeal. Proceedings of the Institution of mechanical Ingineers 1 (1961) 33-40.

[11] S.W.E. Earles, C.K. Lee, Instabilities arising from the frictional interaction of a Pin-Disc System resulting in noise generation. Transactions of the American Society of Mechanical Engineers, Journal of engineering for Industry 1 (1976) 81-86. 
[12] S. Earles, P. Chambers, Disc brake squeal noise generation: Predicting its dependency on system parameters including damping. International Journal of Vehicle Design (1987) 8 538552.

[13] B. Herve, J.-J. Sinou, H. Mahe, L. Jezequel, Analysis of squeal noise and mode coupling instabilities including damping and gyroscopic effects. European Journal of Mechanics, A/Solids 27 (2008) 141-160.

[14] S.S. Antoniou, A. Cameron, C.R. Gentle, The Friction-speed relation from stick-slip data. Wear 36 (1976) 235-254.

[15] J.T. Oden, J.A.C. Martins, Models and computational methods for dynamic friction phenomena. Computer Methods in Applied mechanics and Engineering 52 (1985) 527-634.

[16] F. Van De Velde, P. De Baets, A new approach of stick-slip based on quasi-harmonic tangential oscillations. Wear 216 (1998) 15-26.

[17] F. Van De Velde, P. De Baets, The relation between friction force and relative speed during the slip-phase of stick-slip cycle. Wear 219 (1998) 220-226.

[18] N. Kinkaid, O. O'Reilly, P. Papadopoulos, Automotive disc brake squeal. Journal of Sound and Vibration 267 (2003) 105-166.

[19] H. Ouyang, W. Nack, Y. Yuan, F. Chen, Numerical analysis of automotive disc brake squeal: a review, International Journal of vehicle Noise and Vibration 1 (2005) 207-231.

[20] N. Millner, An Analysis of Disc Brake Squeal. SAE Technical Paper 780332 (1978).

[21] A.F. D’Souza, A.H. Dweib, Self-excited vibration induced by dry friction. Part ii: Stability and limit-cycle analysis. Journal of Sound and Vibration 137 (1990) 177-190. 
[22] M. Eriksson, S. Jacobson, Friction behaviour and squeal generation of disc brakes at low speeds. In proceedings of the Institution of mechanical Engineers, 215 (2001), 1245-1256.

[23] N. Hoffmann, L. Gaul, Effects of damping on mode-coupling instability in frictioninduced oscillations. Zeitschrift FUR Angewandte Mathematik und Mechanik 83 (2003) 524534.

[24] K. Shin, J.E.Oh, M.J. Brennan, Nonlinear analysis of friction induced vibrations of a two degree of freedom model for disc brake squeal noise. JSME International Journal 45 (2002) 426-432.

[25] K. Shin, M.J. Brennan, J.E. Oh, C.J. Harris, Analysis of disc brake noise using a twodegree-of-freedom model. Journal of Sound and Vibration 254 (2002), 837-848.

[26] J.-J.Sinou, L. Jezequel, Mode coupling instability in friction-induced vibrations and its dependency on system parameters including damping. European Journal of Mechanics A/Solid 26 (2007) 107-122.

[27] N. Coudeyras, J.-J. Sinou, S. Nacivet, A new treatment for predicting the self-excited vibrations of nonlinear systems with frictional interfaces: the CHBM. Journal of Sound and Vibration 319 (2008) 1175-1199.

[28] B.T. Polyak, R. Tempo, Probabilistic robust design with linear quadratic regulator. Systems \& Control Letters 43 (2001) 343-353.

[29] R.F. Stengel, Some effects of parameter variations on the latteral-directional stability of aircraft. AIAA Journal of Guidance and Control 3 (1980) 124-131.

[30] R.F. Stengel, L. Ryan, Stochastic robustness of linear time-invariant control systems. IEEE Transactions on Automatic Control 36 (1991) 82-87. 
[31] Q.Wang, R.F. Stengel, Probabilistic Control of Nonlinear Uncertainty Dynamic Systems, in: G. Calafiore and F. Dabbene ( Eds.), 2006 Springer

[32] N. Wiener, The homogeneous chaos. American Journal of Mathematics. 60 (1938) 897936.

[33] R. G. Ghanem, P. D. Spanos, Stochastic Finite Elements: a Spectral Approach, Springer Verlag 199, revised Ed.

[34] S.S. Isukapalli, A. Roy, P.G. Georgopoulos, Stochastic response surface methods (SRSMs) for uncertainty propagation: application to environmental and biological systems. Risk Analysis 18 (1998) 351-363.

[35] A. Sandu, C. Sandu, M. Ahmadian, Modeling multibody dynamic systems with uncertainties. Part I: Numerical application. Multibody system Dynamic 15 (2006) 369-391.

[36] C. Sandu, A. Sandu, M. Ahmadian, Modeling multibody dynamic systems with uncertainties. Part II: Theoretical and computational aspects. Multibody system Dynamic 15 (2006) 241-262.

[37] D. Xiu, G.E. Karniadakis, Modeling uncertainty in steady state diffusion problems via generalized polynomial chaos, Computer Methods in Applied Mechanics and Engineering 191 (2002) 4927-4948.

[38] D. Xiu, G.E. Karniadakis, Modelling uncertainty in flow simulations via generalized polynomial chaos. Journal of Computational Physics 187 (2003) 137-167.

[39] T. Crestaux , O. Le Maitre, J.M. Martinez, Polynomial Chaos Expansion for Sensitivity Analysis. Reliability Engineering and system safety, 94 (2009) 1161-1172. 
[40] B. Sudret, Global sensitivity analysis using polynomial chaos expansions. Reliability Engineering and system safety 93 (2007) 964-979.

[41] L. Nechak, S. Berger, E. Aubry, Robust analysis of uncertain dynamic systems: combination of the centre manifold and polynomial chaos theories. WSEAS Transaction on Systems 9 (2010) 386-395.

[42] E. Blanchard, A. Sandu, A., C. Sandu, Polynomial chaos-based parameter estimation methods applied to vehicle system. Proceeding of the Institution of Mechanical Engineers Part K: J. of Multi-Body Dynamics 224 (2010) 59-81.

[43] E. Blanchard, A. Sandu, C. Sandu, Parameter estimation for mechanical systems via an explicit representation of uncertainty. International Journal for Computer-Aided Engineering Computation. 26 (2009), 541-569.

[44] A.H.C. Smith, A. Monti, F. Ponci, Indirect measurements via a polynomial chaos observer. IEEE Transaction on Instrumentation and Measurement 56 (2007) 743-752.

[45] F.S. Hover, S. Michael. Triantafyllou, Application of polynomial chaos in stability and control. Automatica 42 (2006) $789-795$

[46] Z.K. Nagy, R.D. Braatz, Distributional uncertainty analysis using power series and polynomial chaos expansions. Journal of Process Control 17 (2006) 229-240.

[47] P.S. Beran, C.L. Pettit, D.L. Millman, Uncertainty quantification of limit-cycle oscillations. Journal of Computational Physics 217 (2006) 217-247.

[48] C.L. Pettit, P.S. Beran, Spectral and multi-resolution Wiener expansions of oscillatory stochastic process. Journal of Sound and Vibration 294 (2006) 752-779 
[49] J. Fisher, R. Bhattacharya, Stability analysis of stochastic systems using polynomial chaos, American Control Conference, 2008, pp. 4250-4255.

[50] L. Nechak, S. Berger, E. Aubry, A polynomial chaos approach to the robust analysis of the dynamic behavior of friction systems. European Journal of Mechanics A/Solids 30 (2011) 594-607.

[51] A.H. Nayfeh, B. Balachandran, Applied nonlinear dynamics, Wiley-Interscience Publication, New York, 1995.

[52] J. Hultén, Drum brake squeal- a self exciting mechanism with constant friction, in: SAE. Truck and Bus Meeting, Dettroit, 1993.

[53] J.-J. Sinou, G. Fritz, 1. Jezequel, The Role of Damping and Definition of the Robust Damping Factor for a Self-Exciting Mechanism with Constant Friction. Journal of Vibration and Acoustics 129 (2007) 297-307 
